Sharif University of Technology
Scientia Iranica
SCIENTIA $\quad \begin{gathered}\text { Transactions D: Computer Science ES Engineering and Electrical Engineering } \\ \text { http://scientiairanica.sharif.edu }\end{gathered}$

\title{
Tuning the implementable structures of fractional-order PID controllers for control of FOPDT processes
}

\author{
M. Ashjaee and M.S. Tavazoei* \\ Department of Electrical Engineering, Sharif University of Technology, Tehran, Iran.
}

Received 2 September 2018; received in revised form 10 July 2019; accepted 21 December 2019

\author{
KEYWORDS \\ Optimal tuning; \\ Implementable \\ fractional-order PID \\ controller; \\ Integer-order \\ approximation; \\ Optimization; \\ ISE performance \\ index; \\ ISTE performance \\ index.
}

\begin{abstract}
This study presents a set of rules for optimal tuning of a class of integer-order controllers, known as implementable fractional-order PID controllers, so that they can be employed to control First Order Plus Dead Time (FOPDT) processes. To this end, "tuning based on the implementable form of the controller" is an approach that has been applied instead of the common approach of "tuning based on the ideal form of the controller". Consequently, no contradiction is found between the behavior of the tuned controller and that of the implemented controller. Also, algebraic relations between the values of cost functions, which are defined based on Integral Square Error (ISE) and Integral Square Time Error (ISTE) performance indices and free parameters of the implementable controller are established. Tuning implementable fractional-order PID controllers via the proposed rules guarantees that the values of performance indices are reduced in comparison with the case of using optimal PID controllers. In addition to numerical results, experimental results are also provided to demonstrate the effectiveness of the proposed tuning rules in practical applications.
\end{abstract}

(C) 2022 Sharif University of Technology. All rights reserved.

\section{Introduction}

Over the last years, researchers have turned their attention to the applications of fractional calculus in the control systems engineering by constituting the fractionalorder control field. This field includes the applications of fractional-order differentiation/integration operators to modeling of real-world processes and proposition of effective control laws. Fractional operators, on the one hand, provide a framework for more exact modeling of the processes from different areas such as

\footnotetext{
*. Corresponding author. Tel.: +98 2166164386 ; Fax: +982166023261

E-mail addresses: mehrdadashjaee@gmail.com (M. Ashjaee); tavazoei@sharif.edu (M.S. Tavazoei)
}

electrical engineering [1,2], mechanical engineering [35], medicine [6], and relaxation processes [7] with fewer parameters than integer-order models. On the other hand, their unique characteristics are used to design controllers that are more robust to process variations than traditional integer-order controllers. Some of the most applicable samples of fixed-structure fractionalorder controllers are Fractional Order PD (FOPD), Fractional Order PI (FOPI), and Fractional Order PID (FOPID) controllers, which were originally introduced by Podlubny [8].

The traditional PID controllers represent the most applicable types of the industrial controllers so far. Some reasons for the widespread use of these controllers in industrial applications include the simplicity of structure, existing simple tuning procedures, and robustness against parameter uncertainties. On the other hand, in recent years, the design of five- 
parameter FOPID controllers has drawn many interests amongst researchers [8-11], because they are more flexible than traditional PID controllers and can improve the robustness of the closed-loop system against parameter uncertainties [12]. This is the reason why the studies about the tuning of these controllers for controlling the industrial and experimental processes have been on the rise [13-16]. Up to now, tuning of FOPID controllers based on certain performance criteria in the time domain such as Integral Square Error (ISE), Integral Absolute Error (IAE), Integral Time Absolute Error (ITAE), and Integral Square Time Error (ISTE) or in the frequency domain such as gain crossover frequency and phase margin specifications [17-19] remain the most popular subjects in the area of fractional-order control systems. Therefore, finding an analytical method to solve the optimization problems resulting from satisfying the abovementioned objectives may be laborious (particularly in the cases where we encounter high-dimensional and multi-objective optimization problems). Accordingly, various heuristic algorithms can be employed to solve these complex optimization problems. Several optimization algorithms including Artificial Bee Colony (ABC) algorithm [20,21], Genetic Algorithm (GA) [22,23], Particle Swarm Optimization (PSO) [24], and Tabu Search Algorithm (TSA) [25] have been used for optimal selection of the free parameters of FOPID controllers hitherto. For instance, the authors in [21] designed a FOPID controller to minimize the values of IAE, ISE, and ITAE performance indices by using ABC algorithm. Also, upon applying the GA, in [22], a set of tuning rules was proposed for both integer-order and FOPID controllers in order to optimally control integral and unstable processes based on the IAE criterion. Their results pointed to the higher performance of the systems controlled by FOPID controller than the same ones controlled by standard PID. Similarly, in [23], a novel Adaptive Genetic Algorithm (AGA) was introduced to design a FOPID controller on the basis of a multi-objective optimization task. As another example, in the paper [24] that aimed at tuning the parameters of FOPID controllers, the enhanced PSO algorithm was applied in conjunction with minimizing a cost function defined as a weighted combination of the ITAE and the control effort. Also, there are different tuning methods for FOPID controllers obtained by mathematical optimizations. For example, in [26], a Linear Quadratic Regulator (LQR) based FOPID controller was adopted to control the timedelay fractional-order processes with only one fractional operator by means of Pareto optimization tradeoff solutions. Furthermore, the study [12] proposed a set of algebraic rules for tuning the fractional-order based integer-order controller by considering the ISE performance index. In the proposed methods, in order to implement the fractional controllers in practice, the integer-order approximation of the fractional derivatives and integrators is used because the exact implementation of fractional-order operators in online applications is not possible due to their infinite memory characteristics [27]. This approximation may cause a difference between the behavior of the implemented system and the expected behavior of the closed-loop system [28]. Therefore, the use of an appropriate approximation is necessary for proper implementation of fractional-order operators.

The main purpose of this study is to concentrate on providing a set of tuning rules for the implementable form of FOPID controller by using the algebraic formulation of ISE and ISTE cost functions in terms of free parameters of the fractional-based controller. In doing so, firstly, integer-order realization of the FOPID controller, which is referred to as an implementable form, is obtained. The First Order Plus Dead Time (FOPDT) process is assumed as the system under control in a unity negative feedback structure. Also, the implementable controller and the optimally tuned controller have the same structures. Consequently, no contradiction comes among the specifications of the closed-loop system obtained by theoretical evaluations and the same ones obtained in practice. Moreover, in order to eliminate the error in the computation of ISE and ISTE, an exact analytical procedure is applied while the decrement of them is guaranteed by using the steepest descent algorithm. It is worth noting that a considerable decrement in the cost function value is possible by utilizing this procedure after only a few stages of steepest descent algorithm. Hence, it is shown that the use of FOPID controller allows improving the performance indices.

The organization of the paper is as follows. In Section 2, first, the structures of the process and controller are introduced. Then, the implementable form of the FOPID controller is obtained after normalizing the transfer function of the process and approximating fractional operators in the ideal form of the controller. Section 3 presents algebraic relations for analytically evaluating the ISE and ISTE cost functions. In Section 4, the method of optimal tuning of the free parameters of implementable FOPID controller is described and the resulting tuning rules are given. Next, in Section 5, to verify the efficiency of the proposed tuning rules, some numerical examples as well as an experimental example are given. Finally, Section 6 deals with drawing the main conclusions of the paper.

\section{The models of process and controller}

\subsection{Process and ideal form of the controller}

Figure 1 shows the typical structure of a closed-loop system with unity negative feedback. In this struc- 


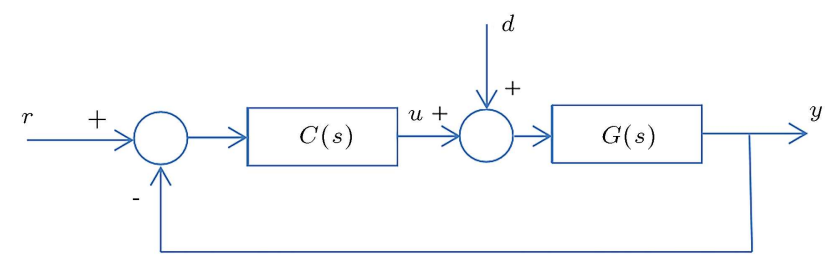

Figure 1. Block diagram of the closed-loop system.

ture, $G(s)$ and $C(s)$ denote the process and controller models, respectively. In this paper, the process is considered in the form of a FOPDT system with the model:

$$
G(s)=\frac{K e^{-\tau s}}{1+T s}
$$

in which $K$ is the steady-state gain, $\tau$ the deadtime, and $T$ the positive time-constant. Several step response-based techniques have been known for approximating the process dynamics by using an FOPDT transfer function [29]. The primary form of the considered controller is described by transfer function [8]:

$$
C_{F}(s)=k_{p}+\frac{k_{i}}{s^{\lambda}}+k_{d} s^{\mu}, \quad \lambda, \mu \in \mathbb{R}^{+} .
$$

The FOPID $\left(P I^{\lambda} D^{\mu}\right)$ controller in Eq. (2) has five free parameters for tuning: $k_{p}, k_{i}$, and $k_{d}$ are constant coefficients; $\lambda$ and $\mu$ are the fractional orders of integration and derivation terms, respectively. In comparison to use of the traditional PID controller, the extra freedom caused by $\lambda$ and $\mu$ in the FOPID controller helps improve the performance of the control system. In this paper, to simplify the optimal tuning rules, orders $\lambda$ and $\mu$ are assumed as follows:

$$
\lambda=1+\nu, \quad \mu=1-\nu
$$

It is clear that with $\nu=0$, the classical form of the PID controller is obtained.

\subsection{The integer-order approximation of controller}

Using integer-order approximations of fractional-order controller is common in practice because the ideal forms of these controllers have infinite memory characteristics [27]. However, when a fractional-order controller is replaced by its integer-order approximation, there is no guarantee for meeting the intended control objectives. Therefore, an intermediate design scheme, as shown in the diagram of Figure 2, is used for optimal tuning of the free parameters. By using this scheme, first, a parameterized fixed-structure integerorder approximation of the fractional-order controller is obtained. Then, optimal tuning rules are proposed to obtain a suboptimal controller [28]. In this approach, there is no divergence between the ideal behavior of the controller and the behavior of its implementable form. Many of the available studies about the optimal tuning of the fractional-order controllers have made use of this design paradigm, indirectly $[12,21,22,24,28]$. In addition, the use of this approach for optimal tuning of the fractional-order controllers creates a condition in which the frequency domain constraints and specifications agree with the expected theoretical values of them in practice [28]. By using the above-mentioned intermediate design approach, the fractional structure is used as a simplified map for tuning a high-order controller (In this case, the number of tuning parameters is equal to that of free parameters of the fractional structure, whereas the implemented controller is a high-order one) [28]. There are many approximation methods in discrete-time or frequency domain for approximating the fractional-order operators with integer-order linear time-invariant filters [30,31]. For example, some of the approximation techniques in the discrete-time domain are based on Power Series Expansion (PSE) and Continued Fraction Expansion (CFE) methods. Also, several methods including Carlson's, Matsuda's, and Oustaloup's methods exist for the integer-order continuous model approximation of fractional-order operators. One of the most generally used techniques is Oustaloup's recursive approximation method in which the fractional-order differentiator is formulated by a series of the rational functions as follows [30]:

$$
s^{\nu}=k_{e} \prod_{i=1}^{N} \frac{1+\left(\frac{s}{\omega_{z, i}}\right)}{1+\left(\frac{s}{\omega_{p, i}}\right)}, \quad 0<\nu<1 .
$$

The poles and zeros are calculated by using the following recursive rules:

$$
\begin{aligned}
& \eta=\left(\frac{\omega_{H}}{\omega_{L}}\right)^{\frac{1-\nu}{N}}, \quad \kappa=\left(\frac{\omega_{H}}{\omega_{L}}\right)^{\frac{\nu}{N}}, \\
& \omega_{z, 1}=\sqrt{\eta} \omega_{L}, \\
& \omega_{p, i}=\kappa \omega_{z, i}, \quad i=1, \cdots, N
\end{aligned}
$$

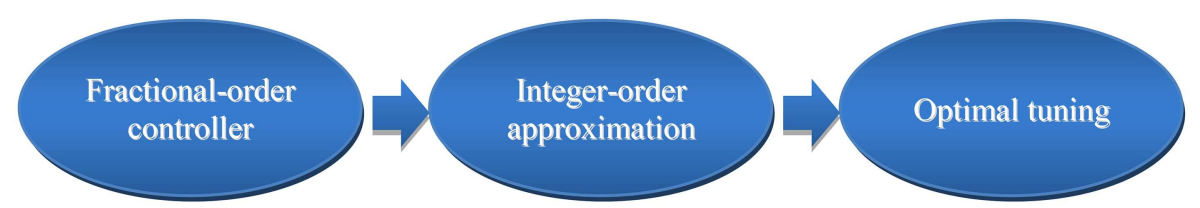

Figure 2. An intermediate design scheme for tuning the implementable structures of fractional-order controllers. 


$$
\omega_{z, i+1}=\eta \omega_{p, i}, \quad i=1, \cdots, N-1 .
$$

The integer-order approximation of $s^{\nu}$ is only valid in the boundary of low and high cut-off frequencies $\left[\omega_{L}, \omega_{H}\right]$. Also, $N$ denotes the number of poles and zeros that should be chosen in advance and determines the order of approximating integer-order filter. Thus, to approximate the ideal controller in Eq. (2) with the orders given in Eq. (3), it is enough to replace the term $s^{\nu}$ by an integer-order filter. With the order $N=2$ and the frequency range $[0.1,1000] \mathrm{rad} / \mathrm{s}$, a parameterized integer-order approximation of the term $s^{\nu}$ is gained. Upon substituting the corresponding terms in Eq. (2) with this filter, the following integer-order controller:

$$
\begin{aligned}
C_{I}(s)=k_{p} & +\frac{k_{i}}{k_{e}}\left(\frac{\left(1+10^{-\nu} s\right)\left(1+10^{-\nu-2} s\right)}{s\left(1+10^{\nu} s\right)\left(1+10^{\nu-2} s\right)}\right) \\
& +\frac{k_{d}}{k_{e}}\left(\frac{s\left(1+10^{-\nu} s\right)\left(1+10^{-\nu-2} s\right)}{\left(1+10^{\nu} s\right)\left(1+10^{\nu-2} s\right)}\right),
\end{aligned}
$$

is obtained, where:

$$
k_{e}=\frac{\left(1+10^{-\nu}\right)\left(1+10^{-\nu-2}\right)}{\left(1+10^{\nu}\right)\left(1+10^{\nu-2}\right)} .
$$

\subsection{Normalization}

In this section, for normalizing the process transfer function $(G(s))$ in Eq. (1), first, an open-loop transfer function of the control system, drawn in Figure 1, is obtained as follows:

$$
G_{o l}(s)=\left(\frac{K e^{-\tau s}}{1+T s}\right)\left(k_{p}+\frac{k_{i}}{s^{\lambda}}+k_{d} s^{\mu}\right),
$$

where $C_{F}(s)$ is considered as the controller. By use of the variable transformation $\xi=T s$, the open-loop transfer function in Eq. (6) can be rewritten in the $\xi$ domain as follows:

$$
\bar{G}_{o l}(\xi)=\left(\frac{K e^{-\frac{\tau}{T} \xi}}{1+\xi}\right)\left(k_{p}+\frac{k_{i} T^{\lambda}}{\xi^{\lambda}}+\frac{k_{d}}{T^{\mu}} \xi^{\mu}\right) .
$$

Therefore, the free parameters of the resultant controller can be optimally tuned for each FOPDT process scaled by ratio $\frac{\tau}{T}$, which is called the normalized dead time. The normalized FOPDT process and the normalized fractional-order controller have the following models:

$$
\bar{G}(\xi)=\frac{e^{-\frac{\tau}{T} \xi}}{1+\xi}
$$

and:

$$
\bar{C}_{F}(\xi)=\bar{k}_{p}+\frac{\bar{k}_{i}}{\xi^{\lambda}}+\bar{k}_{d} \xi^{\mu}
$$

respectively. Afterwards, the normalized fractionalorder controller in Eq. (8) is approximated, as given in Eq. (5). This procedure yields the normalized implementable controller:

$$
\begin{aligned}
\bar{C}_{I}(\xi)=\bar{k}_{p} & +\frac{\bar{k}_{i}}{k_{e}}\left(\frac{\left(1+10^{-\nu} \xi\right)\left(1+10^{-\nu-2} \xi\right)}{\xi\left(1+10^{\nu} \xi\right)\left(1+10^{\nu-2} \xi\right)}\right) \\
& +\frac{\bar{k}_{d}}{k_{e}}\left(\frac{\xi\left(1+10^{-\nu} \xi\right)\left(1+10^{-\nu-2} \xi\right)}{\left(1+10^{\nu} \xi\right)\left(1+10^{\nu-2} \xi\right)}\right) .
\end{aligned}
$$

Thus, it is enough that the free parameters $\bar{k}_{p}, \bar{k}_{i}, \bar{k}_{d}$, and $\nu$ in Eq. (9) be optimally tuned for the normalized process given in Eq. (7) at different values of $\frac{\tau}{T}$ based on the performance criteria. Now, by reusing the variable transformation $s=\frac{\xi}{T}$ for $\bar{C}_{I}(\xi) \bar{G}(\xi)$, the implementable fractional-order controller is derived in the following form:

$$
\begin{aligned}
C(s)=k_{p} & +\frac{k_{i}}{k_{e}}\left(\frac{\left(1+10^{-\nu} T s\right)\left(1+10^{-\nu-2} T s\right)}{s\left(1+10^{\nu} T s\right)\left(1+10^{\nu-2} T s\right)}\right) \\
& +\frac{k_{d}}{k_{e}}\left(\frac{s\left(1+10^{-\nu} T s\right)\left(1+10^{-\nu-2} T s\right)}{\left(1+10^{\nu} T s\right)\left(1+10^{\nu-2} T s\right)}\right)
\end{aligned}
$$

The coefficients $k_{p}, k_{i}$, and $k_{d}$ are optimally achievable for each FOPDT process by utilizing the optimal values of $\bar{k}_{p}, \bar{k}_{i}$, and $\bar{k}_{d}$ in the case of the normalized integerorder controller, given in Eq. (9), through the following relations:

$$
k_{p}=\frac{\bar{k}_{p}}{K}, \quad k_{i}=\frac{\bar{k}_{i}}{K T}, \quad k_{d}=\frac{\bar{k}_{d} T}{K} .
$$

In this paper, the implementable fractional-order controller $(C(s))$ with the transfer function Eq. (10) is tuned for each FOPDT process given in Eq. (1). Consequently, the problem of incompatibility between the theoretical specifications of the closed-loop system and the specifications of its implementable form is removed because the implemented controller is the same as the optimally tuned fractional-order based controller.

\section{Analytical evaluation of cost functions}

\subsection{Analytical calculation of ISE}

In the optimal control theory, to satisfy the intended control objective by the closed-loop system in the time domain or the frequency domain, a cost function is usually considered, which should be minimized by properly choosing the free parameters of the controller. In this paper, one of the performance criteria adopted for the optimization task is the ISE performance index. Using this index as the cost function in the design of control system makes the step response of the closed-loop system more rapid [32]. The ISE index is formulated as follows:

$$
J=\int_{0}^{\infty} e^{2}(t) d t
$$

where $e(t)$ (with the Laplace transform $E(s)$ ) is the error of the closed-loop system to the unit step reference input, i.e.: 


$$
e(t)=1-y(t), \quad t \geq 0 .
$$

It is clear that the Laplace transform of the error of time-delay systems contains exponential term $\left(e^{-\tau s}\right)$ that corresponds to the dead time of the process. In previous studies, different techniques for evaluation of ISE in such cases have been utilized in which the approximation methods play essential roles. For example, the authors in [21] used the sampled values of the error $(e(t))$ during a certain interval to calculate the integral in Eq. (12). Moreover, in [12,33], the exponential term of $E(s)$ was substituted by a rational function by applying the Pade approximation method. These techniques are not entirely accurate for calculating the ISE performance index due to the application of approximation methods. Here, the ISE cost function is evaluated by an analytical procedure in which no approximation technique has been used. Let $E(s)$ be given by:

$$
E(s)=\frac{\hat{B}(s)+\hat{D}(s) e^{-\tau s}}{\hat{A}(s)+\hat{C}(s) e^{-\tau s}},
$$

where $\hat{A}(s), \hat{B}(s), \hat{C}(s)$, and $\hat{D}(s)$ are real polynomials. The assumption is that the integral of Eq. (12) exists or, equivalently, the closed-loop system is stable. Of note, a necessary, but not sufficient, condition for stability of the system is that the poles of $E(s)$ lie in the open left-half of $s$-plane [34]. The application of the Parseval's theorem results in the following integral:

$$
J=\frac{1}{2 \pi j} \int_{-j \infty}^{+j \infty} E(s) E(-s) d s .
$$

By substituting $E(s)$ in Eq. (14), calculation of ISE is possible using contour integration such that only a finite number of related poles should be analyzed [35]. Supposing that the integrals around the semicircles at infinity are zero, the integral $J$ is evaluated as follows:

$$
\begin{aligned}
J= & -\sum_{k} \operatorname{res}_{s=s_{k}}\left(\frac{\hat{B}(s)+\hat{D}(s) e^{-\tau s}}{\hat{A}(s)+\hat{C}(s) e^{-\tau s}}\right) \\
& \left(\frac{\hat{B}(-s) \hat{A}(s)-\hat{D}(-s) \hat{C}(s)}{\hat{A}(-s) \hat{A}(s)-\hat{C}(-s) \hat{C}(s)}\right)
\end{aligned}
$$

where the summation is taken over all the zeros $\left(s_{k}\right)$ of the following equation:

$$
\hat{A}(-s) \hat{A}(s)-\hat{C}(-s) \hat{C}(s)=0 .
$$

Here, by applying the mentioned analytical procedure, the exact value of the ISE performance index is calculated. To this end, first, the Laplace transform of the error signal for a unit step reference input is determined. For the closed-loop system shown in Figure 1, $E(s)$ is in the form of:

$$
E(s)=\frac{1}{s(1+G(s) C(s))} .
$$

Here, the transfer function $E(s)$ is rewritten as in Eq. (13), where:

$$
\begin{aligned}
\hat{A}(s)= & k_{e} s(1+T s)\left(1+10^{\nu} T s\right)\left(1+10^{\nu-2} T s\right), \\
\hat{B}(s)= & k_{e}(1+T s)\left(1+10^{\nu} T s\right)\left(1+10^{\nu-2} T s\right), \\
\hat{C}(s)= & K k_{p} k_{e} s\left(1+10^{\nu} T s\right)\left(1+10^{\nu-2} T s\right) \\
& +K k_{i}\left(1+10^{-\nu} T s\right)\left(1+10^{-2-\nu} T s\right) \\
& +K k_{d} s^{2}\left(1+10^{-\nu} T s\right)\left(1+10^{-2-\nu} T s\right), \\
\hat{D}(s)= & 0 .
\end{aligned}
$$

Hence, for exact calculation of ISE, the roots of Eq. (16) should be determined. To this end, the polynomial equation,

$$
a s^{8}+b s^{6}+c s^{4}+d s^{2}+e=0,
$$

should be solved where the coefficients $a, b, c, d$, and $e$ are as follows:

$$
\begin{aligned}
& a=10^{4 \nu-4} T^{6} k_{e}^{2}-a_{1}^{2}, \\
& b=-k_{e}^{2} T^{4}\left(10^{2 \nu}+10^{2 \nu-4}+10^{4 \nu-4}\right)+b_{1}^{2}-2 a_{1} c_{1} \\
& c=k_{e}^{2} T^{2}\left(10^{2 \nu}+10^{2 \nu-4}+1\right)+2 b_{1} d_{1}-2 a_{1} e_{1}-c_{1}^{2} \\
& d=-k_{e}^{2}+d_{1}^{2}-2 c_{1} e_{1} \\
& e=-e_{1}^{2}
\end{aligned}
$$

with:

$$
\begin{aligned}
& a_{1}=10^{-2 \nu-2} K k_{d} T^{2} \\
& b_{1}=K\left(10^{2 \nu-2} k_{p} k_{e} T^{2}+k_{d} T\left(10^{-\nu}+10^{-\nu-2}\right)\right), \\
& c_{1}=K\left(k_{p} k_{e} T\left(10^{\nu}+10^{\nu-2}\right)+10^{-2 \nu-2} k_{i} T^{2}+k_{d}\right), \\
& d_{1}=K\left(k_{p} k_{e}+k_{i} T\left(10^{-\nu}+10^{-\nu-2}\right)\right) \\
& e_{1}=K k_{i} .
\end{aligned}
$$

Hence, the coefficients of Eq. (18) are some functions of the parameters of the process and controller. The degree of polynomial in Eq. (18) is eight where the coefficients of odd power terms of $s$ in this polynomial are zero. Therefore, the change in variable $x=s^{2}$ results in the fourth-order equation:

$$
a x^{4}+b x^{3}+c x^{2}+d x+e=0 .
$$

Quartic equations are the highest degree polynomials that can be analytically solved by radicals in which no iterative technique needs to be utilized [36]. Accordingly, the polynomial Eq. (20) can be analytically solved. This means that the roots of Eq. (20) may be expressed according to the coefficients $a, b, c, d$, 
and $e$, which are the functions of parameters of the process and controller. Subsequently, considering the process parameters, roots of Eq. (18) are the functions of controller parameters $k_{p}, k_{i}, k_{d}$, and $\nu$. In order to compute the ISE criterion via Relation (15), the residue of the function in the root $s_{i}$ is first evaluated as follows [37]:

$$
\begin{aligned}
J_{i}=- & \left(\frac{\hat{B}\left(s_{i}\right)+\hat{D}\left(s_{i}\right) e^{-\tau s_{i}}}{\hat{A}\left(s_{i}\right)+\hat{C}\left(s_{i}\right) e^{-\tau s_{i}}}\right) \\
& \left(\frac{\hat{B}\left(-s_{i}\right) \hat{A}\left(s_{i}\right)-\hat{D}\left(-s_{i}\right) \hat{C}\left(s_{i}\right)}{\hat{E}_{i}\left(s_{i}\right)}\right),
\end{aligned}
$$

where $\hat{E}_{i}\left(s_{i}\right)$ is the factorized form of the polynomial in Eq. (18):

$$
\hat{E}_{i}\left(s_{i}\right)=a \prod_{\substack{k=1 \\ k \neq i}}^{8}\left(s_{i}-s_{k}\right)
$$

Accordingly, by the sum of $J_{i}$ 's in each root of Eq. (18), an algebraic relation with respect to controller parameters used for computation of the ISE is derived as follows:

$$
J=J\left(k_{p}, k_{i}, k_{d}, \nu\right) .
$$

Hence, the ISE performance index can be exactly measured by substituting the values of controller parameters in the cost function $J$. This result brings about simplicity in searching for a minimum value of $J$ and high accuracy in the optimal tuning of controller parameters.

\subsection{Analytical calculation of ISTE}

In this section, the method of analytical evaluation of another cost function, which is assumed as ISTE performance index, is described. This index is mathematically defined as follows:

$$
J=\int_{0}^{\infty} t^{2} e^{2}(t) d t
$$

where $e(t)$ is the error of the closed-loop system to a unit step reference input. Taking into account that in the control system under study, as represented in Eq. (17), $\hat{D}(s)=0$ and using the procedure proposed in [38], which is similar to the approach utilized for the ISE index evaluation, the following formula is obtained for calculating the ISTE criterion:

$$
\begin{gathered}
J=\sum_{k} \operatorname{res}_{s_{k}}\left(\frac{\hat{N}(s)}{(\hat{A}(-s) \hat{A}(s)-\hat{C}(-s) \hat{C}(s))^{3}}\right) \\
\left(\frac{\hat{B}(-s)}{\hat{A}(-s)+\hat{C}(-s) e^{\tau s}}\right)
\end{gathered}
$$

where:

$$
\begin{aligned}
N_{21}(s)= & N_{11}^{\prime}(s) \hat{A}(s)-2 N_{11}(s) \hat{A}^{\prime}(s), \\
N_{22}(s)= & N_{11}^{\prime}(s) \hat{C}(s)+\left(N_{12}^{\prime}(s)-\tau N_{12}(s)\right) \hat{A}(s) \\
& -2 N_{12}(s) \hat{A}^{\prime}(s)-2 N_{11}(s)\left(\hat{C}^{\prime}(s)-\tau \hat{C}(s)\right), \\
N_{23}(s)= & \left(N_{12}^{\prime}(s)-\tau N_{12}(s)\right) \hat{C}(s) \\
& -2 N_{12}(s)\left(\hat{C}^{\prime}(s)-\tau \hat{C}(s)\right), \\
N_{11}(s)= & \hat{B}^{\prime}(s) \hat{A}(s)-\hat{A}^{\prime}(s) \hat{B}(s), \\
N_{12}(s)= & \hat{B}^{\prime}(s) \hat{C}(s)-\hat{B}(s) \hat{C^{\prime}}(s)+\tau \hat{B}(s) \hat{C}(s),
\end{aligned}
$$

where the superscript $\left({ }^{\prime}\right)$ denotes derivative with respect to $s$ and the sum of residues is calculated at the roots of the following equation:

$$
(\hat{A}(-s) \hat{A}(s)-\hat{C}(-s) \hat{C}(s))^{3}=0 .
$$

Eq. (26) is written as follows:

$$
\left(a s^{8}+b s^{6}+c s^{4}+d s^{2}+e\right)^{3}=0,
$$

with the parameters defined in Eq. (19). Hence, the roots of Eq. (26) are the same as those of Eq. (18) at iteration 3. Based on the residue theory [37], the following formula is established for the evaluation of residue at the $i$ th root of Eq. (27):

$$
J_{i}=\lim _{s \rightarrow s_{i}} \frac{1}{2} \frac{\partial^{2}}{\partial s^{2}}\left(\left(\frac{\hat{N}(s)}{\hat{E}_{i}^{3}(s)}\right)\left(\frac{\hat{B}(-s)}{\hat{A}(-s)+\hat{C}(-s) e^{\tau s}}\right)\right),
$$

where $\hat{E}_{i}(s)$ has the same form as that presented in Eq. (22). Similar to the ISE cost function, with the sum of $J_{i}$ 's for each root of Eq. (27) and by considering process parameters $(K, \tau, T)$, an algebraic relation is formed to evaluate the integral in Eq. (24) in terms of free parameters of the controller. Therefore, by substituting the values of controller parameters into the function of Eq. (28) for each root of the polynomial of Eq. (27), the exact value of the ISTE performance index is calculated.

\section{Procedure of optimal tuning}

In this section, an optimization method based on the steepest descent algorithm is applied in order to tune the implementable FOPID controller in Eq. (10). The vector of the controller parameters is given by:

$$
P=\left[\begin{array}{c}
k_{p} \\
k_{i} \\
k_{d} \\
\nu
\end{array}\right],
$$

which will be optimally tuned to minimize the cost 
function $J$ for the FOPDT processes. The steepest descent algorithm (also called gradient or Cauchy's method) is one of the line search algorithms for scalar unconstrained optimization [12]. At each stage of the algorithm, the gradient of the objective function $\left(\nabla J\left(P^{i}\right)\right)$ and the step length $\left(\alpha^{i}\right)$ need to be computed and inserted in the following formula [39]:

$$
P^{i+1}=P^{i}-\alpha^{i} \nabla J\left(P^{i}\right), \quad \alpha^{i} \in \mathbb{R}^{+} .
$$

The ideal choice for step length is derived from the one-dimensional optimization problem as follows:

$$
\min _{\alpha^{i}} J\left(P^{i}-\alpha^{i} \nabla J\left(P^{i}\right)\right), \quad \alpha^{i}>0,
$$

and solving this problem may not be a simple task. Here, to find a local minimizer, the following equation:

$$
\frac{d J\left(P^{i}-\alpha^{i} \nabla J\left(P^{i}\right)\right)}{d \alpha^{i}}=0
$$

is considered, where $\alpha^{i}$ is approximately determined using a numerical method at each iteration. As demonstrated in the previous section, the fact that the ISE and ISTE performance indices are functions of free parameters of the controller makes it possible to plot the cost functions according to the step length. For instance, in Figure 3, a typical plot of the ISE cost function with respect to $\alpha$ has been drawn. In this special case, using Eq. (31), the optimal value of step length is almost $\alpha \approx 0.4$. Therefore, the local minimization $\alpha$ may be numerically estimated based on Eq. (31) at each iteration.

It is clear that the integer-order PID controller is indeed a special case of Eq. (10) in which the fractionalorder $\nu$ is set to zero. Hence, the initial point for the parameter vector given in Eq. (29) may be chosen as follows:

$$
P^{0}=\left[\begin{array}{c}
k_{p}^{\text {ord }} \\
k_{i}^{\text {ord }} \\
k_{d}^{\text {ord }} \\
0
\end{array}\right],
$$

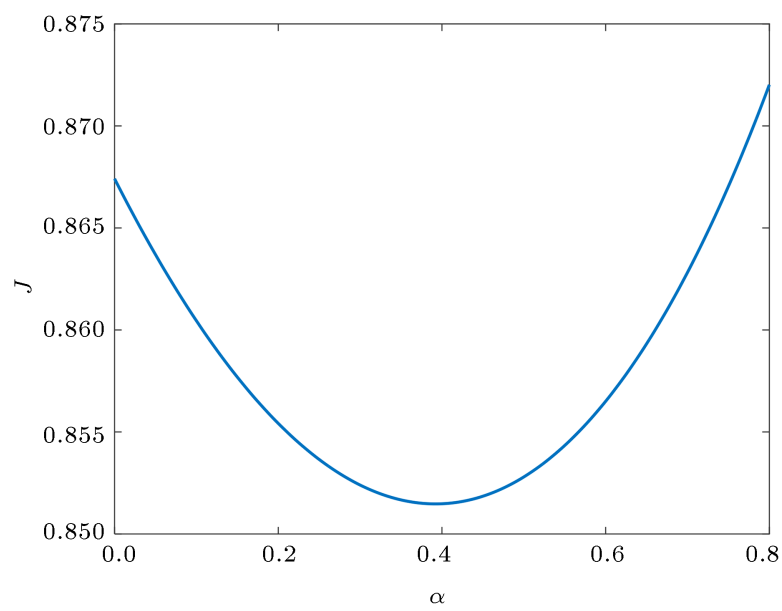

Figure 3. A typical ISE cost function with respect to $\alpha$. where $k_{p}^{\text {ord }}, k_{i}^{\text {ord }}$, and $k_{d}^{\text {ord }}$ are considered as the optimal parameters of the ordinary PID controller.

\subsection{Analytical gradients of the cost functions}

As explained in the previous subsection, the gradient of the objective function with respect to the controller parameters defined by:

$$
\nabla J(P)=\left[\begin{array}{c}
\frac{\partial J}{\partial k_{p}} \\
\frac{\partial J}{\partial k_{i}} \\
\frac{\partial J}{\partial k_{d}} \\
\frac{\partial J}{\partial \nu}
\end{array}\right]=\sum_{i=1}^{8} \nabla J_{i}(P),
$$

should be calculated at each stage of the steepest descent algorithm. As presented in Section 3, the ISE and ISTE performance criteria are expressed in terms of $k_{p}, k_{i}, k_{d}$, and $\nu$. Accordingly, the gradient of the cost functions can be analytically evaluated. Hence, by applying the chain rule to differentiate the objective functions, the exact value of the gradient is obtained as follows [40]:

$$
\nabla J_{i}(P)=\frac{\partial J_{i}}{\partial s_{i}} \nabla s_{i}(P)+\left[\begin{array}{l}
\frac{\partial J_{i}}{\partial k_{p}}\left(P, s_{i}\right) \\
\frac{\partial J_{i}}{\partial k_{i}}\left(P, s_{i}\right) \\
\frac{\partial J_{i}}{\partial k_{d}}\left(P, s_{i}\right) \\
\frac{\partial J_{i}}{\partial \nu}\left(P, s_{i}\right)
\end{array}\right],
$$

where $\nabla s_{i}(P)$ denotes the gradient of the $i$ th root of Eq. (18) with respect to the vector of controller parameters. $\nabla s_{i}(P)$ is calculated by applying the chain rule again via the following formula:

$$
\begin{aligned}
\nabla s_{i}(P)= & \frac{\partial s_{i}}{\partial a} \nabla a(P)+\frac{\partial s_{i}}{\partial b} \nabla b(P)+\frac{\partial s_{i}}{\partial c} \nabla c(P) \\
& +\frac{\partial s_{i}}{\partial d} \nabla d(P)+\frac{\partial s_{i}}{\partial e} \nabla e(P) .
\end{aligned}
$$

Therefore, the gradients of ISE and ISTE performance indices are computed using the algebraic and accurate relations. After substituting the gradient $\nabla J(P)$ into Eq. (30), the optimal value of step length is evaluated based on Eq. (31).

\subsection{Optimal tuning rules}

To find the optimal tuning rules for the free parameters of the implementable fractional-order controller in Eq. (10), first, the normalized form of the controller given in Eq. (9) is optimally tuned for the normalized transfer function in Eq. (7) with different ratios of $\frac{\tau}{T} \in$ $[0.1,2]$. The interpolated plots of optimal parameters of the normalized implementable controller $\left(\bar{k}_{p}, \bar{k}_{i}\right.$, $\bar{k}_{d}$, and $\nu$ ) are drawn in Figures 4 and 5 based on 


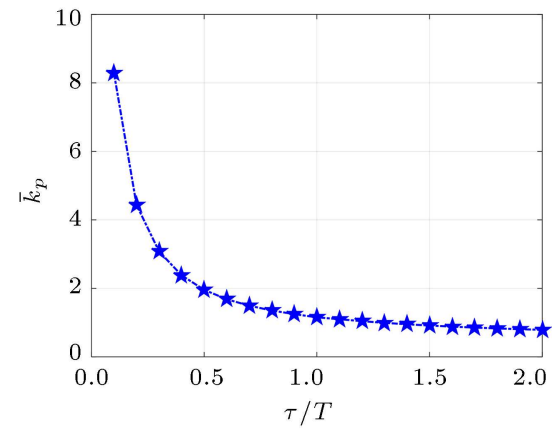

(a) $\bar{k}_{p}$

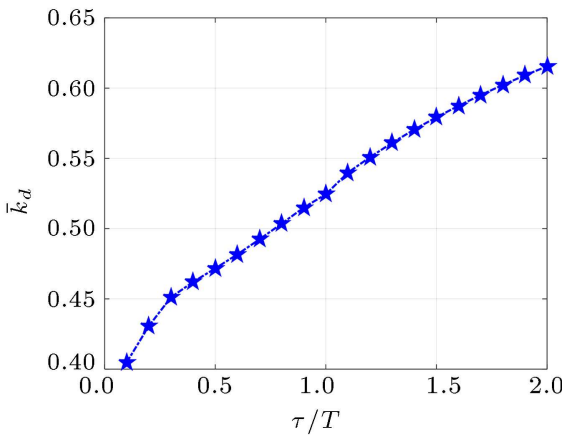

(c) $\bar{k}_{d}$

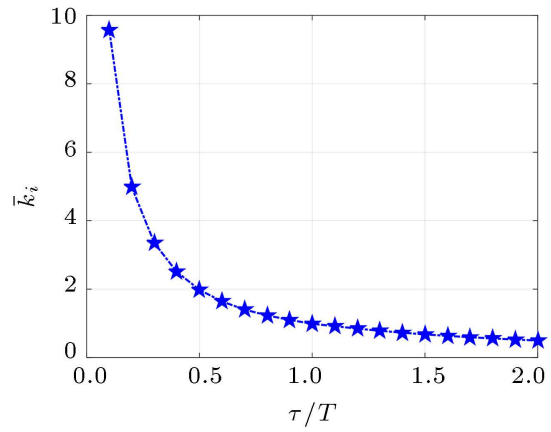

(b) $\bar{k}_{i}$

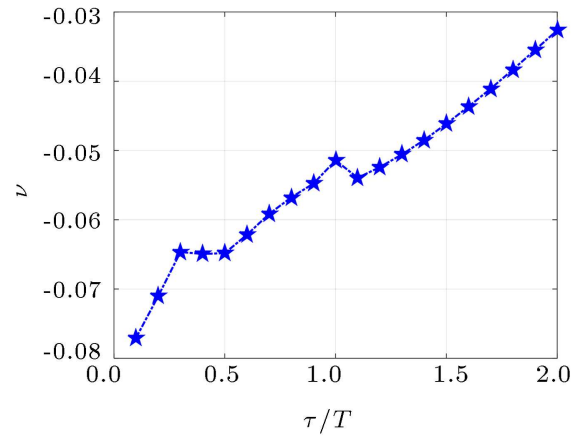

(d) $\nu$

Figure 4. Optimal parameters of the normalized implementable controller based on the ISE performance criterion.

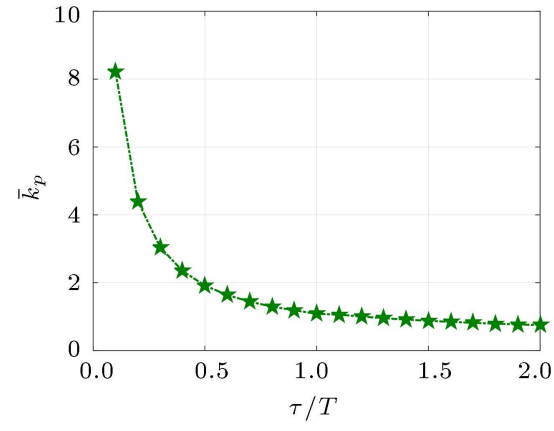

(a) $\bar{k}_{p}$

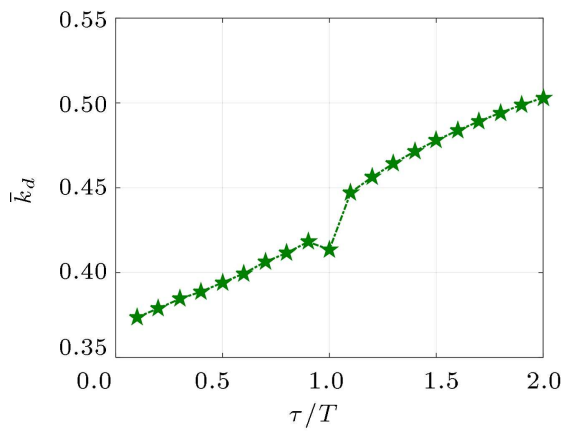

(c) $\bar{k}_{d}$

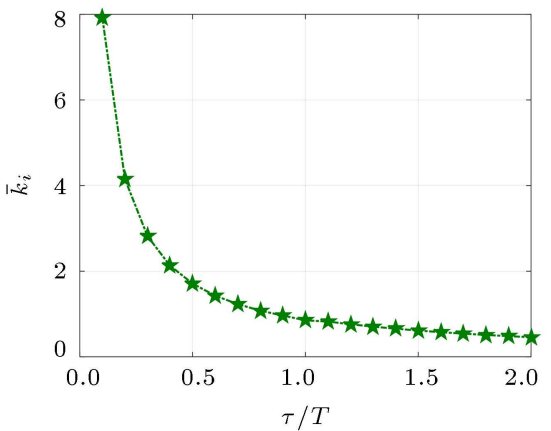

(b) $\bar{k}_{i}$

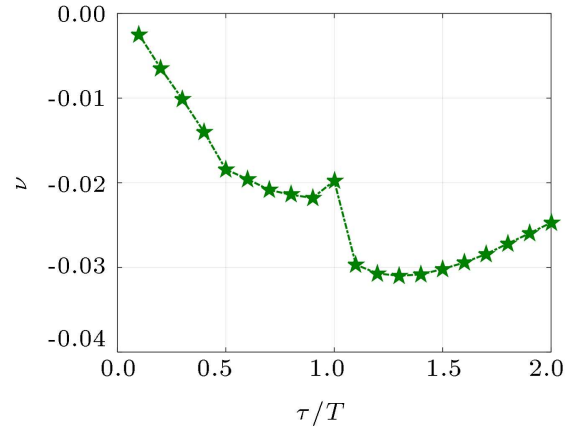

(d) $\nu$

Figure 5. Optimal parameters of the normalized implementable controller based on the ISTE performance criterion.

the ISE and ISTE performance criteria, respectively. To this end, eight stages of the proposed algorithm in the previous section have been repeated and the optimal parameters of ordinary PID controller tuned based on ISE and ISTE cost functions, similar to that proposed in [41], have been assumed as the starting points. By using least squares fitting method [42], the normalized parameters are formulated according to $\frac{\tau}{T}$ with separate coefficients at the subintervals $[0.1$, $1]$ and $[1.1,2]$. Then, by applying the relations in 
Table 1. Values of the coefficients of $k_{p}$ in Eq. (34).

\begin{tabular}{cccccccc}
\hline Range of $\frac{\boldsymbol{\tau}}{\boldsymbol{T}}$ & Performance index & $\boldsymbol{a}_{\boldsymbol{p}}$ & $\boldsymbol{b}_{\boldsymbol{p}}$ & $\boldsymbol{c}_{\boldsymbol{p}}$ & $\boldsymbol{d}_{\boldsymbol{p}}$ & $\boldsymbol{e}_{\boldsymbol{p}}$ & $\boldsymbol{f}_{\boldsymbol{p}}$ \\
\hline \multirow{2}{\tau}{$\in[0.1,1]$} & ISE & 1.03 & -0.9049 & -0.02914 & 0.16 & 0 & 0 \\
& ISTE & 1.135 & -0.8727 & 0 & 0 & -0.2266 & -0.2665 \\
& & & & & & & \\
$\frac{\tau}{T} \in[1.1,2]$ & ISE & 1.139 & -0.7034 & -0.007517 & 0.03746 & 0 & 0 \\
& ISTE & 0.7627 & -0.9779 & 0 & 0 & 0 & 0.3657 \\
\hline
\end{tabular}

Table 2. Values of the coefficients of $k_{i}$ in Eq. (34).

\begin{tabular}{cccccccc}
\hline Range of $\frac{\boldsymbol{\tau}}{\boldsymbol{T}}$ & Performance index & $\boldsymbol{a}_{\boldsymbol{i}}$ & $\boldsymbol{b}_{\boldsymbol{i}}$ & $\boldsymbol{c}_{\boldsymbol{i}}$ & $\boldsymbol{d}_{\boldsymbol{i}}$ & $\boldsymbol{e}_{\boldsymbol{i}}$ & $\boldsymbol{f}_{\boldsymbol{i}}$ \\
\hline \multirow{2}{\tau}{$\in[0.1,1]$} & ISE & 1.195 & -0.9084 & -0.6795 & 1.646 & -1.172 & 0 \\
& ISTE & 1.046 & -0.8935 & 0 & 0 & 0.09235 & -0.2772 \\
& & & & & & & \\
$\frac{\tau}{T} \in[1.1,2]$ & ISE & 1.016 & -0.925 & -0.00061 & -0.00856 & 0.00093 & 0 \\
& ISTE & 1.104 & -0.7354 & 0 & 0 & 0 & -0.2061 \\
\hline
\end{tabular}

Table 3. Values of the coefficients of $k_{d}$ in Eq. (34).

\begin{tabular}{cccccccc}
\hline Range of $\frac{\tau}{\boldsymbol{T}}$ & Performance index & $\boldsymbol{P}_{\mathbf{0}}$ & $\boldsymbol{P}_{\mathbf{1}}$ & $\boldsymbol{P}_{\mathbf{2}}$ & $\boldsymbol{P}_{\mathbf{3}}$ & $\boldsymbol{P}_{\mathbf{4}}$ & $\boldsymbol{P}_{\mathbf{5}}$ \\
\hline \multirow{2}{\tau}{$\in[0.1,1]$} & ISE & 0.3624 & 0.5137 & -1.032 & 1.093 & -0.413 & 0 \\
& ISTE & 0.3722 & -0.02178 & 0.486 & -1.363 & 1.667 & -0.7273 \\
& & & & & & & \\
$\frac{\tau}{T} \in[1.1,2]$ & ISE & 0.342 & 0.2605 & -0.08733 & 0.012773 & 0 & 0 \\
& ISTE & 0.3653 & -0.1426 & 0.5124 & -0.4387 & 0.1639 & -0.0231 \\
\hline
\end{tabular}

Table 4. Values of the coefficients of $\nu$ in Eq. (34).

\begin{tabular}{|c|c|c|c|c|c|c|c|c|}
\hline Range of $\frac{\tau}{T}$ & Performance index & $P_{0}$ & $P_{1}$ & $P_{2}$ & $P_{3}$ & $P_{4}$ & $P_{5}$ & $P_{6}$ \\
\hline \multirow{2}{*}{$\frac{\tau}{T} \in[0.1,1]$} & ISE & -0.06944 & -0.2542 & 2.549 & -9.162 & 15.52 & -12.46 & 3.829 \\
\hline & ISTE & -0.007727 & -0.1751 & 1.032 & -3.615 & 6.276 & -5.184 & 1.638 \\
\hline \multirow{2}{*}{$\frac{\tau}{T} \in[1.1,2]$} & ISE & -0.03511 & -0.06152 & 0.05428 & -0.01411 & 0.00133 & 0 & 0 \\
\hline & ISTE & 0.3967 & -1.38 & 1.859 & -1.342 & 0.5447 & -0.1163 & 0.01009 \\
\hline
\end{tabular}

Eq. (11), the following tuning rules are obtained:

$$
\begin{aligned}
k_{p}= & \frac{1}{K}\left(a_{p}\left(\frac{\tau}{T}\right)^{b_{p}}+c_{p}\left(\frac{\tau}{T}\right)^{3}+d_{p}\left(\frac{\tau}{T}\right)^{2}\right. \\
& \left.+e_{p}\left(\frac{\tau}{T}\right)+f_{p}\right), \\
k_{i}= & \frac{1}{T K}\left(a_{i}\left(\frac{\tau}{T}\right)^{b_{i}}+c_{i}\left(\frac{\tau}{T}\right)^{3}+d_{i}\left(\frac{\tau}{T}\right)^{2}\right. \\
& \left.+e_{i}\left(\frac{\tau}{T}\right)+f_{i}\right), \\
k_{d}= & \frac{T}{K}\left(P_{5}\left(\frac{\tau}{T}\right)^{5}+P_{4}\left(\frac{\tau}{T}\right)^{4}+P_{3}\left(\frac{\tau}{T}\right)^{3}\right. \\
& \left.+P_{2}\left(\frac{\tau}{T}\right)^{2}+P_{1}\left(\frac{\tau}{T}\right)+P_{0}\right),
\end{aligned}
$$

$$
\begin{aligned}
\nu= & P_{6}\left(\frac{\tau}{T}\right)^{6}+P_{5}\left(\frac{\tau}{T}\right)^{5}+P_{4}\left(\frac{\tau}{T}\right)^{4}+P_{3}\left(\frac{\tau}{T}\right)^{3} \\
& +P_{2}\left(\frac{\tau}{T}\right)^{2}+P_{1}\left(\frac{\tau}{T}\right)+P_{0}
\end{aligned}
$$

where the values of coefficients are represented in Tables 1 to 4 for both ISE and ISTE performance criteria. Consequently, to optimally tune the implementable fractional-order controller in Eq. (10) for FOPDT processes based on ISE and ISTE performance indices, it is enough to calculate the parameters given in Eq. (34).

\section{Simulation and experimental results}

In this section, to investigate the effectiveness and performance of the proposed implementable fractionalorder controller with the tuning rules in Eq. (34), three examples (two numerical examples and an experimental example) are presented. In each example, both the implementable FOPID and conventional optimal PID controllers have been designed based on the ISE 
and ISTE performance indices and compared with each other (of note, in the following examples, the sample time used for discretizing the controllers in the Matlab/Simulink environment is chosen as $0.01 \mathrm{~s}$ ).

\subsection{Example 1}

Consider the following process:

$$
G_{1}(s)=\frac{3.13 e^{-5 s}}{1+43.333 s}
$$

For control of the FOPDT process Eq. (35), the free parameters of the implementable FOPID controller with the transfer function of Eq. (10) are tuned by using the ISE-based rules given in Eq. (34) as follows:

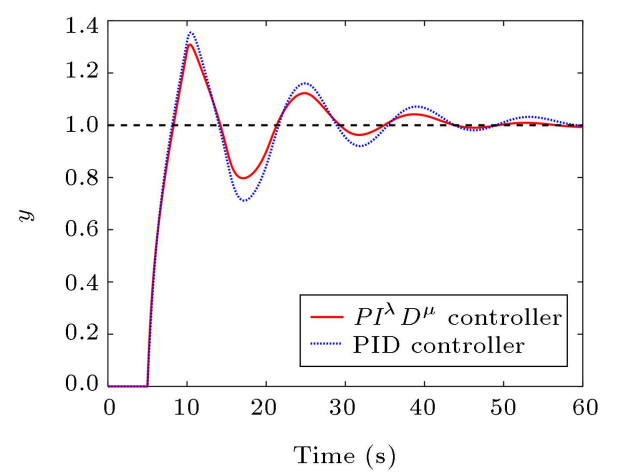

(a) Output signals

$$
P_{1}^{*}=\left[\begin{array}{c}
k_{p_{1}}^{*} \\
k_{i_{1}}^{*} \\
k_{d_{1}}^{*} \\
\nu_{1}^{*}
\end{array}\right]=\left[\begin{array}{c}
2.3231 \\
0.0618 \\
5.6698 \\
-0.0764
\end{array}\right] .
$$

Figure 6 shows the unit step responses of the closedloop system with the implementable FOPID controller tuned by Eq. (36) and optimal PID controller based on the ISE criterion [41]. As compared in Table 5, the implementable FOPID controller outperforms the optimal integer-order PID controller in terms of ISE performance index. Also, as compared to optimal PID control method, the overshoot, peak time, and settling time of the closed-loop step response are reduced by using the implementable FOPID controller, while the

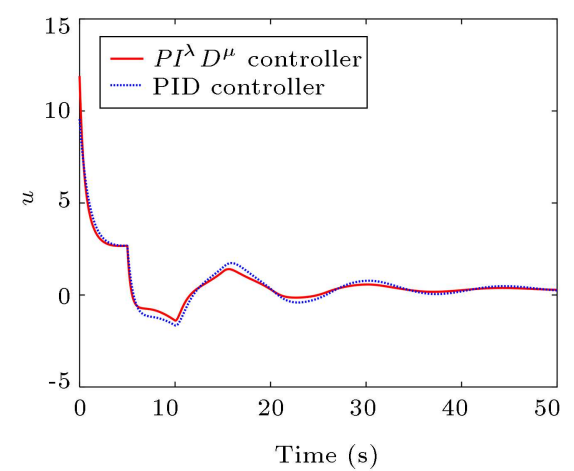

(b) Control signals

Figure 6. Step responses of the closed-loop system of the process Eq. (35) with the controllers tuned based on ISE index.

\begin{tabular}{|c|c|c|c|c|c|c|c|c|}
\hline Process & $\begin{array}{c}\text { Performance } \\
\text { index }\end{array}$ & Controller & $\begin{array}{c}\text { Overshoot } \\
(\%)\end{array}$ & $\begin{array}{c}\text { Peak time } \\
(\mathrm{s})\end{array}$ & $\begin{array}{c}\text { Settling time } \\
(\mathrm{s})\end{array}$ & ISE & ISTE & $\begin{array}{l}\text { RMS } \\
\text { of } u\end{array}$ \\
\hline \multirow{5}{*}{ System 1} & \multirow{2}{*}{ ISE } & PID & 35.40 & 10.50 & 56.03 & 6.46 & - & 0.81 \\
\hline & & FOPID & 30.84 & 10.21 & 42.06 & 6.12 & - & 0.78 \\
\hline & \multirow{2}{*}{ ISTE } & PID & 24.63 & 10.93 & 36.24 & - & 156.31 & 0.70 \\
\hline & & FOPID & 22.96 & 11.14 & 35.90 & - & 143.44 & 0.68 \\
\hline & IAE & FOPID [43] & 2.76 & 32.05 & 37.02 & 6.70 & 161.9 & 0.62 \\
\hline \multirow{5}{*}{ System 2} & \multirow{2}{*}{ ISE } & PID & 34.89 & 20.89 & 85.79 & 11.95 & - & 0.82 \\
\hline & & FOPID & 35.34 & 20.92 & 63.45 & 11.79 & - & 0.95 \\
\hline & \multirow{2}{*}{ ISTE } & PID & 26.13 & 21.30 & 65.08 & - & 848.42 & 0.80 \\
\hline & & FOPID & 24.13 & 20.95 & 62.86 & - & 734.46 & 0.88 \\
\hline & IAE & FOPID [43] & 1.39 & 58.63 & 42.30 & 13.70 & 1290.22 & 0.69 \\
\hline \multirow{4}{*}{ System 3} & \multirow{2}{*}{ ISE } & PID & 31.25 & 8.7 & 59.8 & 308.83 & - & 4.61 \\
\hline & & FOPID & 27.35 & 8.53 & 39.5 & 270.25 & - & 4.57 \\
\hline & \multirow{2}{*}{ ISTE } & PID & 26.5 & 9.26 & 66.72 & - & 1154.87 & 4.60 \\
\hline & & FOPID & 19.2 & 9.53 & 55.5 & - & 859.69 & 4.58 \\
\hline
\end{tabular}

Table 5. Numerical results related to the representative processes for set-point tracking. 


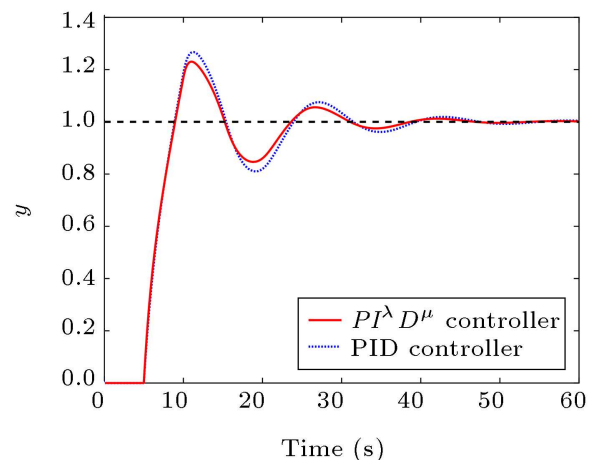

(a) Output signals

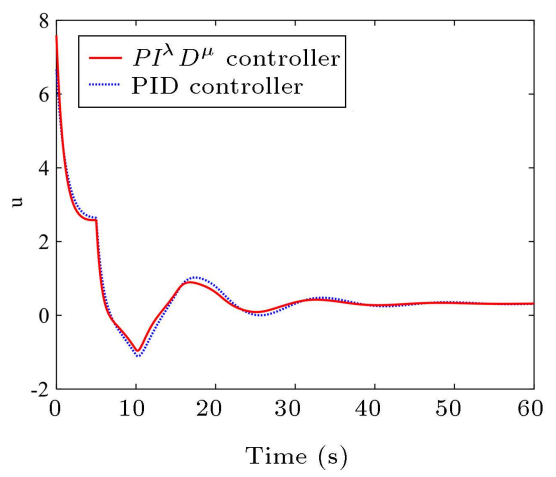

(b) Control signals

Figure 7. Step responses of the closed-loop system of the process Eq. (35) with the controllers tuned based on ISTE index.

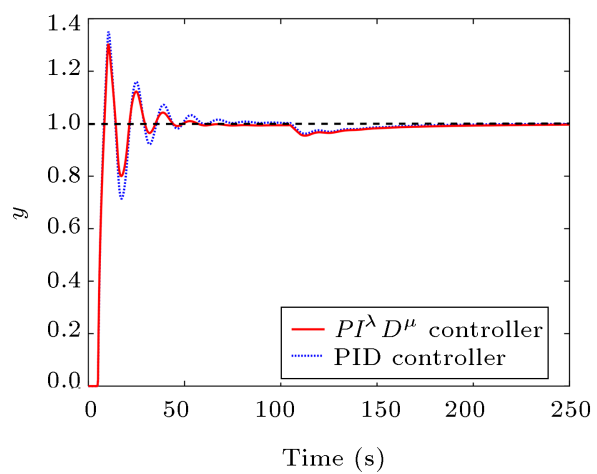

(a) Output signals

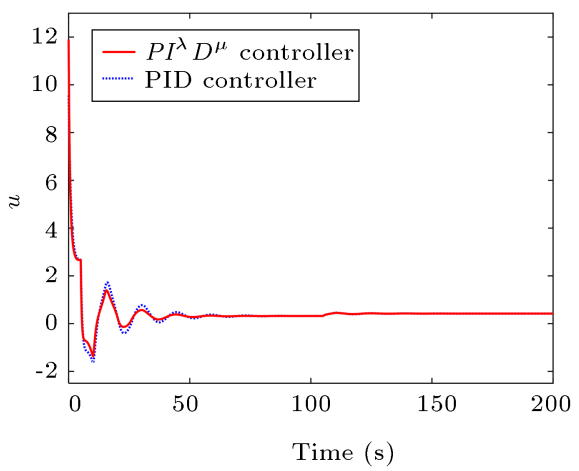

(b) Control signals

Figure 8. Disturbance rejection of the closed-loop system of the process Eq. (35) with the controllers tuned based on ISE index (external disturbance $d=0.1$ has been applied at time $t=100$ ).

Root Mean Square (RMS) values of the control signal a minor increase.

The second comparison is carried out for the feedback control systems designed on the basis of the ISTE performance index. To this end, the conventional PID controller is optimally tuned based on the ISTEbased rules proposed in [41]. The free parameters of the implementable FOPID controller are also adjusted by the formulas in Eq. (34) based on the ISTE criterion, resulting in the following parameter vector for control of process in Eq. (35):

$$
P_{1}^{+}=\left[\begin{array}{c}
k_{p_{1}}^{+} \\
k_{i_{1}}^{+} \\
k_{d_{1}}^{+} \\
\nu_{1}^{+}
\end{array}\right]=\left[\begin{array}{c}
2.2938 \\
0.0511 \\
5.1826 \\
-0.0033
\end{array}\right] .
$$

The output responses and control signals of the closedloop system by using the above-mentioned controllers are depicted in Figure 7 (a) and (b), respectively. From the numerical results shown in Table 5 , a considerable decrement in the value of ISTE index is confirmed due to the use of the implementable FOPID controller instead of the optimal PID controller. Also, as verified in Figures 8 and 9, the performance of the tuned FOPID controllers in external disturbance rejection of step type signals is similar to that of the optimal PID controllers.

\subsection{Example 2}

The following process:

$$
G_{2}(s)=\frac{1.5 e^{-10.3920 s}}{1+8.66 s},
$$

is supposed to be controlled by the implementable FOPID controller tuned based on the ISE and ISTE objective functions. According to the proposed tuning rules, the following parameter vectors:

$$
P_{2}^{*}=\left[\begin{array}{c}
k_{p_{2}}^{*} \\
k_{i_{2}}^{*} \\
k_{d_{2}}^{*} \\
\nu_{2}^{*}
\end{array}\right]=\left[\begin{array}{c}
0.7126 \\
0.0651 \\
3.1802 \\
-0.0524
\end{array}\right],
$$

and:

$$
P_{2}^{+}=\left[\begin{array}{c}
k_{p_{2}}^{+} \\
k_{i_{2}}^{+} \\
k_{d_{2}}^{+} \\
\nu_{2}^{+}
\end{array}\right]=\left[\begin{array}{c}
0.6692 \\
0.0585 \\
2.6346 \\
-0.0311
\end{array}\right],
$$

are assigned to set the implementable FOPID controller according to the ISE and ISTE performance indices, respectively. 


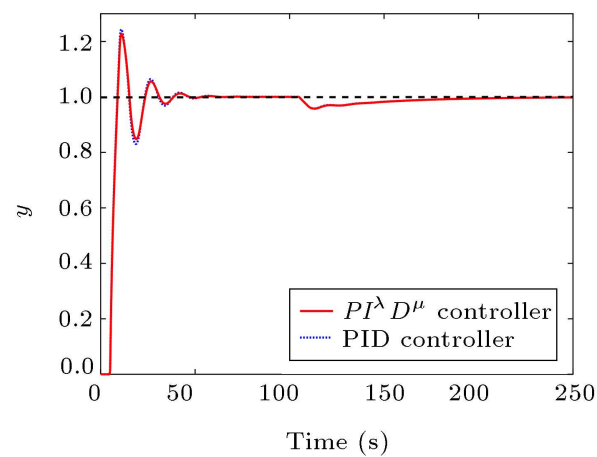

(a) Output signals

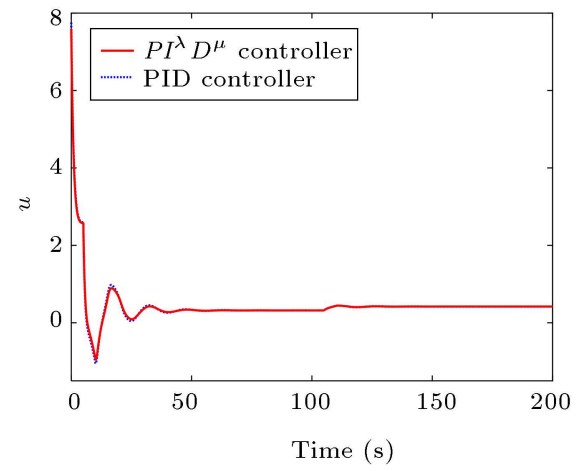

(b) Control signals

Figure 9. Disturbance rejection of the closed-loop system of the process Eq. (35) with the controllers tuned based on ITSE index (external disturbance $d=0.1$ has been applied at time $t=100$ ).

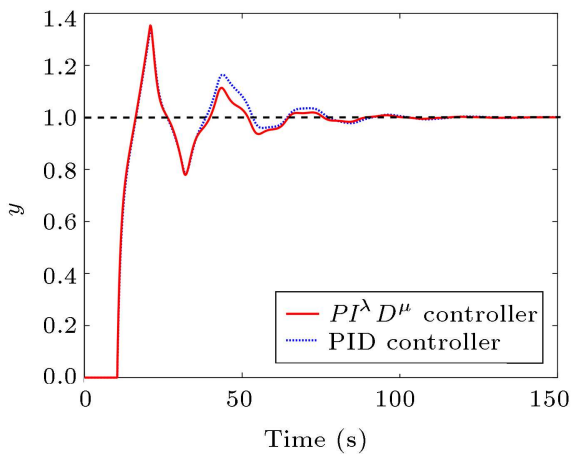

(a) Output signals

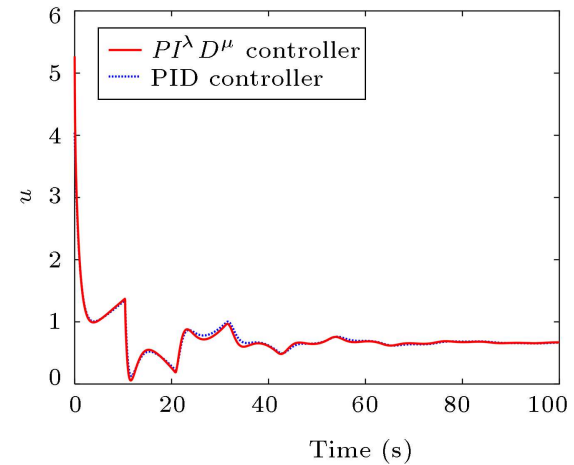

(b) Control signals

Figure 10. Step responses of the closed-loop system of the process Eq. (38) with the controllers tuned based on ISE index.

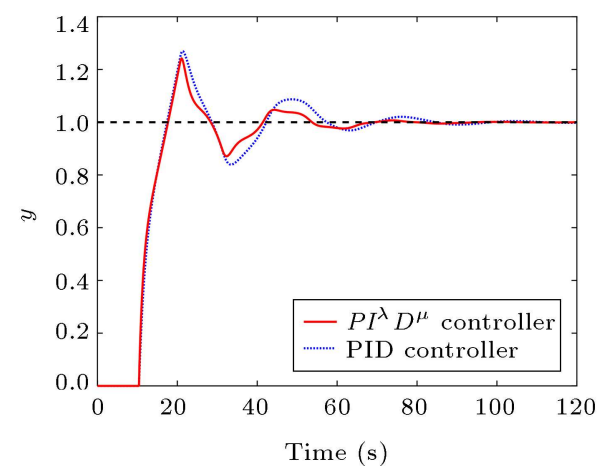

(a) Output signals

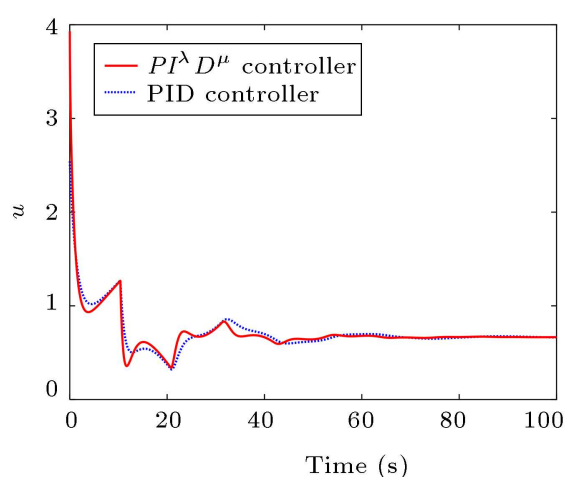

(b) Control signals

Figure 11. Step responses of the closed-loop system of the process Eq. (38) with the controllers tuned based on ISTE index.

Figures 10 and 11 show the set-point step responses of the process $G_{2}(s)$ controlled by the implementable FOPID controllers tuned by the parameters as in Eqs. (39) and (40), respectively, in comparison to those controlled by optimal integer-order PID controllers [41] (the performance of the considered control systems in external disturbance rejection is also compared in Figures 12 and 13). Referring to the results summarized in Table 5 , the superiority of implementable FOPID controller in terms of the ISE and ISTE performance criteria is verified compared to the integer-order PID controller. In the case of ISE, it appears that the overshoot of step response stays almost unchanged, whereas the settling time decreases. Also, for the ISTE case, using the proposed implementable FOPID controller leads to fewer oscillations than an optimal PID controller.

In Table 5, the results obtained from using FOPID controllers tuned via the IAE index-based method introduced in [10,43: Subsection 3.4.5] are also presented. As is expected, the implementable FOPID controller proposed in this study yields lower 


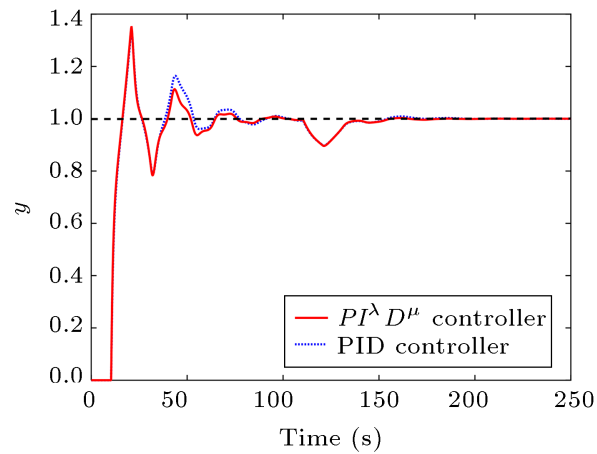

(a) Output signals

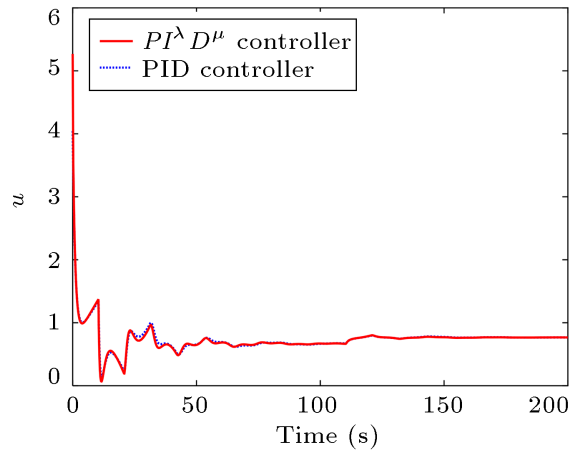

(b) Control signals

Figure 12. Disturbance rejection of the closed-loop system of the process Eq. (38) with the controllers tuned based on ISE index (external disturbance $d=0.1$ has been applied at time $t=100$ ).

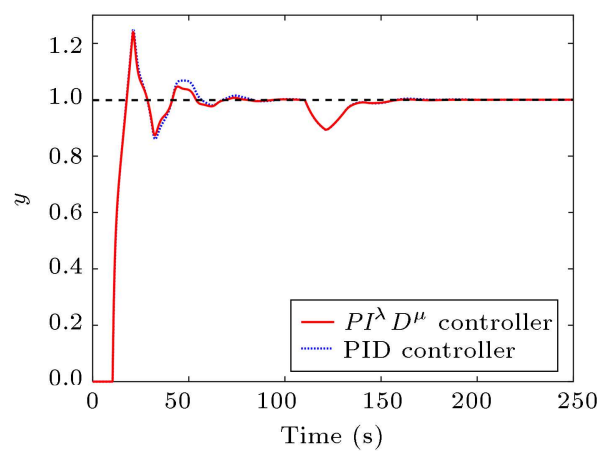

(a) Output signals

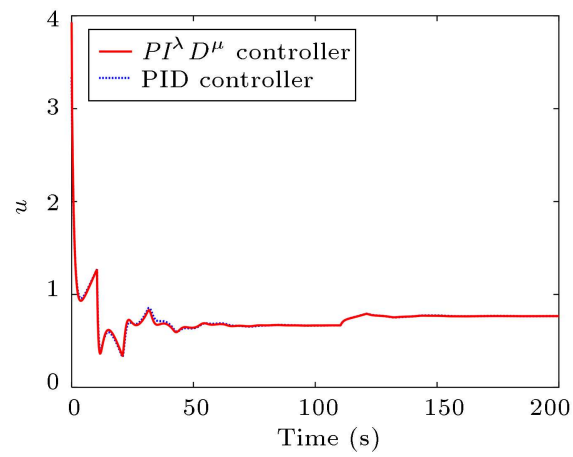

(b) Control signals

Figure 13. Disturbance rejection of the closed-loop system of the process Eq. (38) with the controllers tuned based on ITSE index (external disturbance $d=0.1$ has been applied at time $t=100$ ).

ISE and ISTE values than the case of using FOPID controllers tuned by the method of $[10,43$ : Subsection 3.4.5]. However, due to the nature of the IAE index, the controller tuned on the basis of this criterion results in less maximum overshoot.

\subsection{Example 3: Experimental control of a thermal furnace}

In this example, in order to verify the efficiency of the proposed tuning rules in practical applications, experimental results from the control of a thermal furnace, shown in Figure 14, are presented. For this purpose, first, the FOPDT model of the process from the input voltage to the furnace temperature should be obtained. By using a simple step response test, the transfer function of the process is obtained as follows:

$$
G_{3}(s)=\frac{14.105 e^{-3.6 s}}{1+7.675 s} \text {. }
$$

From the tuning rules of Eq. (34), the free parameters of the implementable FOPID controller for control of process in Eq. (41) are obtained as:

$$
P_{3}^{*}=\left[\begin{array}{c}
k_{p_{3}}^{*} \\
k_{i_{3}}^{*} \\
k_{d_{3}}^{*} \\
\nu_{3}^{*}
\end{array}\right]=\left[\begin{array}{c}
0.1472 \\
0.0196 \\
0.2553 \\
-0.0642
\end{array}\right],
$$

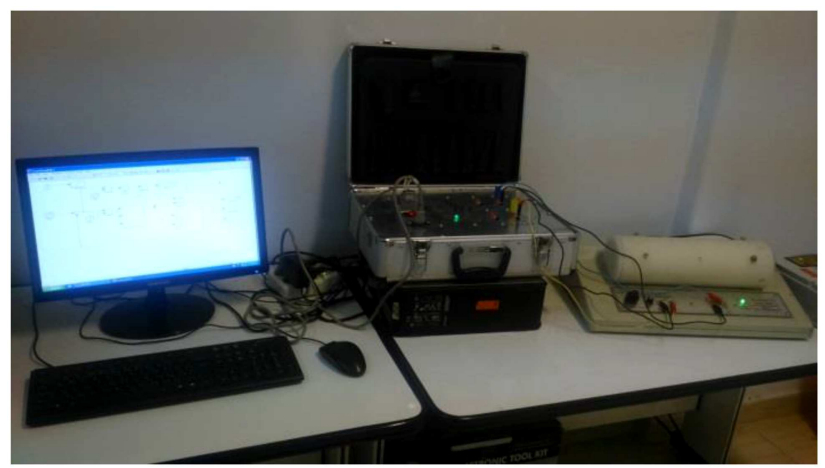

Figure 14. Hardware-in-the-loop thermal furnace control system.

and:

$$
P_{3}^{+}=\left[\begin{array}{c}
k_{p_{3}}^{+} \\
k_{i_{3}}^{+} \\
k_{d_{3}}^{+} \\
\nu_{3}^{+}
\end{array}\right]=\left[\begin{array}{c}
0.1294 \\
0.0168 \\
0.2135 \\
-0.0169
\end{array}\right],
$$

based on the ISE and ISTE performance criteria, respectively. To illustrate the effectiveness of the tuned FOPID controllers, they are compared with the conventional PID controller tuned based on the corresponding cost functions by using the methods presented in [41]. The controllers have been implemented 


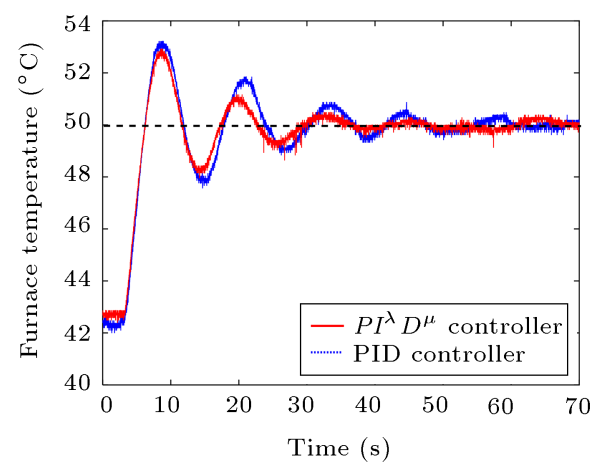

(a) Output signals

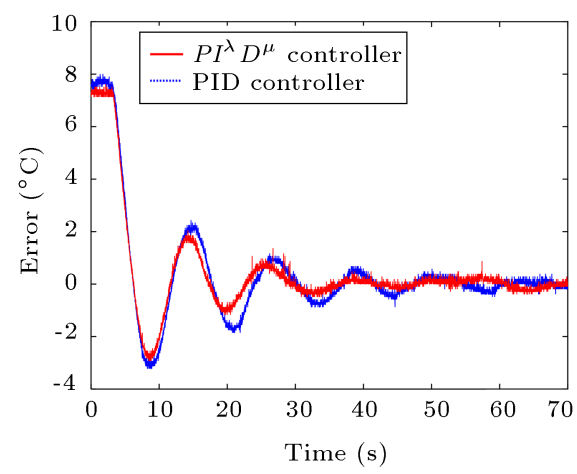

(b) Error signals

Figure 15. Step responses of the thermal furnace controlled by the controllers tuned based on ISE index.

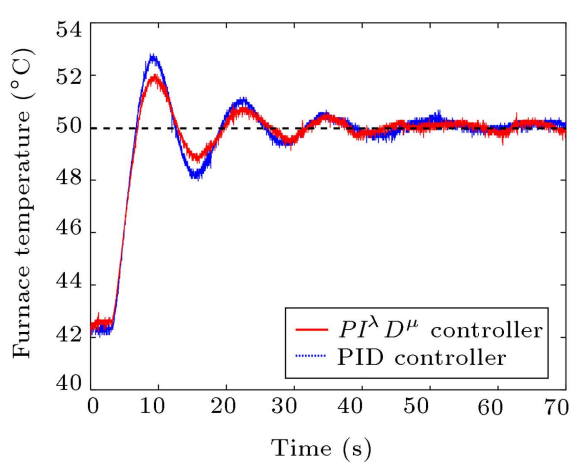

(a) Output signals

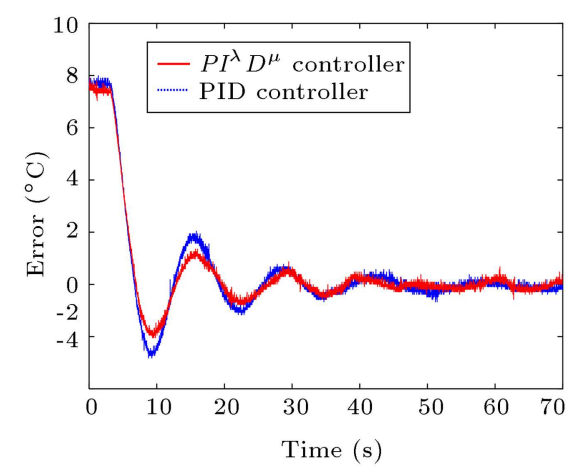

(b) Error signals

Figure 16. Step responses of the thermal furnace controlled by the controllers tuned based on ISTE index.

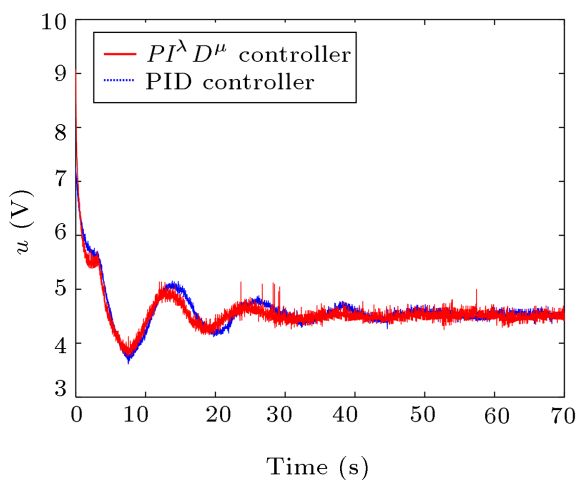

(a) ISE criterion

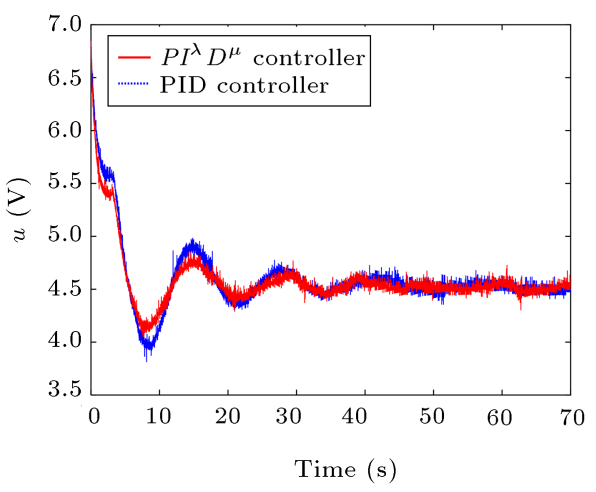

(b) ISTE criterion

Figure 17. Control signals of the closed-loop system of the thermal furnace.

via MATLAB/Simulink Real-Time Workshop and the obtained practical results are presented in Figures 1517.

According to the step responses plotted in Figures 15 and 16 for the thermal furnace controlled by implementable FOPID and integer-order PID controllers and based on the numerical results given in Table 5, it can be seen that the maximum overshoots of the output signals have decreased in the case of using FOPID controller in comparison with the same of integer-order type for both ISE and ISTE criteria. Also, the numerical results depicted in Table 5 verify the improvement of the mentioned cost functions by use of the parameters presented in Eqs. (42) and (43). Furthermore, the comparison of the values of the peak time and settling time reveals that the control system functions faster in the case of using implementable FOPID controllers and the RMS values of the control inputs undergo a little decrease.

\section{Conclusion}

In this study, closed forms for calculating Integral Square Error (ISE) and Integral Square Time Error 
(ISTE) cost functions in the case of using implementable FOPID controllers in control of FOPDT processes were derived. On the basis of these forms, algebraic rules for were proposed tuning the free parameters of FOPID controllers. Based on these rules, it is guaranteed that the resultant performance is superior to that of any conventional PID controller. This finding was confirmed through different numerical and experimental examples.

\section{References}

1. Dzieliński, A., Sarwas, G., and Sierociuk, D. "Comparison and validation of integer and fractional order ultracapacitor models", Advances in Difference Equations, 2011(1), p. 11 (2011).

2. Radwan, A.G., Soliman, A.M., and Elwakil, A.S. "Design equations for fractional-order sinusoidal oscillators: Four practical circuit examples", International Journal of Circuit Theory and Applications, 36(4), pp. 473-492 (2008).

3. Gabano, J.D., Poinot, T., and Kanoun, H. "Identification of a thermal system using continuous linear parameter-varying fractional modelling", IET Control Theory and Applications, 5(7), pp. 889-899 (2011).

4. $\mathrm{Xu}$, J. and Li, J. "Stochastic dynamic response and reliability assessment of controlled structures with fractional derivative model of viscoelastic dampers", Mechanical Systems and Signal Processing, 72, pp. 865-896 (2016).

5. Kumar, S. "A new fractional analytical approach for treatment of a system of physical models using Laplace transform", Scientia Iranica, 21(5), pp. 1693-1699 (2014).

6. Ionescu, C.M. and De Keyser, R. "Relations between fractional-order model parameters and lung pathology in chronic obstructive pulmonary disease", IEEE Transactions on Biomedical Engineering, 56(4), pp. 978-987 (2009).

7. Taghavian, H. and Tavazoei, M.S. "Analytic solution of a system of linear distributed order differential equations in the Reimann-Liouville sense", Scientia Iranica, 27(3), pp. 1384-1397 (2020). DOI: 10.24200/SCI.2018.20335

8. Podlubny, I. "Fractional-order systems and $P I^{\lambda} D^{\mu}$ controllers", IEEE Transactions on Automatic Control, 44(1), pp. 208-214 (1999).

9. Luo, Y. and Chen, Y. "Fractional order [proportional derivative] controller for a class of fractional order systems", Automatica, 45(10), pp. 2446-2450 (2009).

10. Padula, F. and Visioli, A. "Tuning rules for optimal PID and fractional-order PID controllers", Journal of Process Control, 21(1), pp. 69-81 (2011).

11. Fergani, N. and Charef, A. "Process step response based fractional $P I^{\lambda} D^{\mu}$ controller parameters tuning for desired closed loop response", International Journal of Systems Science, 47(3), pp. 521-532 (2016).
12. Rahimian, M.A. and Tavazoei, M.S. "Improving integral square error performance with implementable fractional-order PI controllers", Optimal Control Applications and Methods, 35(3), pp. 303-323 (2014).

13. Monje, C.A., Vinagre, B.M., Feliu, V., et al. "Tuning and auto-tuning of fractional order controllers for industry applications", Control Engineering Practice, 16(7), pp. 798-812 (2008).

14. Li, H., Luo, Y., and Chen, Y. "A fractional order proportional and derivative (FOPD) motion controller: tuning rule and experiments", IEEE Transactions on Control Systems Technology, 18(2), pp. 516-520 (2010).

15. Roy, P. and Roy, B.K. "Fractional order PI control applied to level control in coupled two tank MIMO system with experimental validation", Control Engineering Practice, 48, pp. 119-135 (2016).

16. Khubalkar, S., Chopade, A., Junghare, A., et al. "Design and realization of stand-alone digital fractional order PID controller for buck converter fed DC motor", Circuits, Systems and Signal Processing, 35(6), pp. 2189-2211 (2016).

17. Sayyaf, N. and Tavazoei, M.S. "Robust fractionalorder compensation in the presence of uncertainty in a pole/zero of the plant", IEEE Transactions on Control Systems Technology, 26(3), pp. 797-812 (2018).

18. Sayyaf, N. and Tavazoei, M.S. "Desirably adjusting gain margin, phase margin and corresponding crossover frequencies based on frequency data", IEEE Transactions on Industrial Informatics, 13(5), pp. 2311-2321 (2017).

19. Badri, V. and Tavazoei, M.S. "Some some analytical results on tuning fractional-order [proportionalintegral] controllers for fractional-order systems", IEEE Transactions on Control Systems Technology, 24(3), pp. 1059-1066 (2016).

20. Karaboga, D. and Akay, B. "Proportional-integralderivative controller design by using artificial bee colony, harmony search and the bees algorithms", Proceedings of the Institution of Mechanical Engineers, Part I: Journal of Systems and Control Engineering, 224(7), pp. 869-883 (2010).

21. Kesarkar, A.A. and Selvaganesan, N. "Tuning of optimal fractional-order PID controller using an artificial bee colony algorithm", Systems Science and Control Engineering, 3(1), pp. 99-105 (2015).

22. Padula, F. and Visioli, A. "Optimal tuning rules for proportional-integral-derivative and fractional-order proportional-integral-derivative controllers for integral and unstable processes", IET Control Theory and Applications, 6(6), pp. 776-786 (2012).

23. Chang, L.Y. and Chen, H.C. "Tuning of fractional PID controllers using adaptive genetic algorithm for active magnetic bearing system", WSEAS Transactions on Systems, 8(1), pp. 158-167 (2009). 
24. Cao, J.Y. and Cao, B.G. "Design of fractional order controllers based on particle swarm optimization", $1 S T$ IEEE Conference on Industrial Electronics and Applications, pp. 1-6 (2006).

25. Ateş, A. and Yeroglu, C. "Optimal fractional order PID design via Tabu Search based algorithm", ISA Transactions, 60, pp. 109-118 (2016).

26. Das, S., Pan, I., and Das, S. "Multi-objective LQR with optimum weight selection to design FOPID controllers for delayed fractional order processes", ISA Transactions, 58, pp. 35-49 (2015).

27. Tavazoei, M.S. and Haeri, M. "Rational approximations in the simulation and implementation of fractional-order dynamics: A descriptor system approach", Automatica, 46(1), pp. 94-100 (2010).

28. Rahimian, M.A. and Tavazoei, M.S. "Optimal tuning for fractional-order controllers: an integer-order approximating filter approach", ASME Journal of Dynamic Systems, Measurement and Control, 135(2), 021017 (2013).

29. Kinney, T.B. "Tuning process controllers", Chemical Engineering, 90(19), pp. 67-72 (1983).

30. Podlubny, I., Petraš, I., Vinagre, B.M., et al. "Analogue realizations of fractional-order controllers", Nonlinear Dynamics, 29(1), pp. 281-296 (2002).

31. Machado, J.A. "Delay approximation of fractional integrals", Asian Journal of Control, 15(3), pp. 713722 (2013).

32. Ogata, K., Modern Control Engineering, Prentice Hall of India, New Delhi (1982).

33. Sundaravadivu, K., Arun, B., and Saravanan, K. "Design of fractional order PID controller for liquid level control of spherical tank", IEEE International Conference on Control System, Computing and Engineering (ICCSCE), pp. 291-295 (2011).

34. Walton, K. and Marshall, J.E. "Closed form solution for time delay systems' cost functionals", International Journal of Control, 39(5), pp. 1063-1071 (1984).

35. Kealy, T. and O'Dwyer, A. "Analytical ISE calculation and optimum control system design", In Proceedings of the Irish Signals and Systems Conference, Limerick, Ireland, pp. 418-423 (2003).

36. Shmakov, S.L. "A universal method of solving quartic equations", International Journal of Pure and Applied Mathematics, 71(2), pp. 251-259 (2011).
37. Kreyszig, E. and Norminton, E.J., Advanced Engineering Mathematics, 4th Edn., Wiley, New York (1993).

38. Walton, K., Ireland, B., and Marshall, J.E. "Evaluation of weighted quadratic functional for time-delay systems", International Journal of Control, 44(6), pp. 1491-1498 (1986).

39. Nocedal, J. and Wright, S.J., Numerical Optimization, Springer-Verlag, New York (2006).

40. Courant, R., Differential and Integral Calculus, 2th Edn., John Wiley and Sons (1968).

41. Zhuang, M. and Atherton, D.P. "Automatic tuning of optimum PID controllers", In IEE Proceedings D (Control Theory and Applications), 140(3), pp. 216224 (1993).

42. Nash, J.C., Compact Numerical Methods for Computers: Linear Algebra and Function Minimization, CRC press (1990).

43. Padula, F. and Visioli, A., Advances in Robust Fractional Control, Springer (2015).

\section{Biographies}

Mehrdad Ashjaee received his MSc degree in Electrical Engineering from the Sharif University of Technology, Tehran, Iran in 2017. His current research interests include design of fractional-order PID controllers and integer-order implementation methods for these controllers to be used in real-time applications.

Mohammad Saleh Tavazoei received the BSc, MSc, and $\mathrm{PhD}$ degrees in Electrical Engineering from the Sharif University of Technology, Tehran, Iran in 2003, 2005, and 2008, respectively. He is currently a Full Professor at the Department of Electrical Engineering, Sharif University of Technology. His current research interests include fractional-order circuits and systems and their applications in engineering. He was a recipient of the Young Investigator Award of the IEEE Iran Section in 2012. He was also selected as the Prominent Young Scientist in electrical and computer engineering by the Academy of Sciences of Iran in 2017. 